\section{Retraso de la erupción dental en el síndrome de Down: evaluación crítica de la evidencia}

\section{Delayed tooth eruption in Down syndrome: critical appraisal of evidence}

\section{Resumen}

El síndrome de Down en nińos se asocia con múltiples alteraciones orofaciales, dentro de ellas se ha mencionado el retraso en la erupción dental, cuya comprensión no es aún del todo conocida. Se realizó una revisión para analizar los conceptos de erupción dental y acerca de la evidencia disponible respecto al retraso en la erupción dental de los nińos con síndrome de Down y su relación con la maduración de los dientes o con otros factores sistémicos y locales. Se observó escasez de estudios sobre el tema, sin embargo, con la literatura encontrada se pudo concluir que el retraso de erupción de las piezas dentales parece no tener relación con la maduración de la pieza dental, sino a otros procesos que tienen lugar durante la erupción dental o que puede ser parte del retraso general del crecimiento y del desarrollo que caracteriza al síndrome de Down.

Palabras clave: Erupción dental; Síndrome de Down; Determinación de la edad por los dientes (fuente: DeCS BIREME).

\begin{abstract}
Down syndrome in children is associated with multiple orofacial alterations, among them delayed tooth eruption has been mentioned, the understanding of which is not yet fully known. A review was carried out to analyze the concepts of tooth eruption and the available evidence regarding delayed tooth eruption in children with Down syndrome, and its relationship with tooth maturation or with other systemic and local factors. Few studies were observed on the subject, however, with the found literature it was possible to conclude that the delay in tooth eruption seems not to be related to the maturation of the tooth, but it is related to other processes that take place during tooth eruption or it may be part of the general delay in growth and development that characterizes Down syndrome.
\end{abstract}

Keywords: Tooth eruption; Down Syndrome; Age Determination by Teeth (Source: MeSH NLM).

\section{Artículo de Revisión}

Ursula Ofelia Rivas-Almonte 1,a, Marco Antonio

Cruz-Escalante ${ }^{1, a}$, Carlos Alberto Delgado-Bocanegra ${ }^{2, b}$

${ }^{1}$ Universidad Nacional Mayor de San Marcos, Facultad de Odontología, Lima, Perú.

2 Universidad Nacional Mayor de San Marcos, Facultad de Medicina, Lima, Perú

${ }^{a}$ Magíster. Cirujano Dentista.

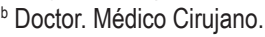

\section{Correspondencia:}

Ursula Ofelia Rivas Almonte: urivasa@unmsm.edu.pe

Calle Cutervo 1818 Cercado de Lima. Lima, Perú. ORCID: 0000-0001-5141-8237

\section{Coautores:}

Marco Antonio Cruz Escalante: marco.cruz1@unmsm. edu.pe

ORCID: 0000-0002-7768-2842

Carlos Alberto Delgado Bocanegra: cdelgadob1@unmsm. edu.pe

ORCID: 0000-0002-6073-8109

\section{Editora:}

Antonieta Pérez-Flores

Universidad de Concepción, Chile

Conflicto de intereses: los autores declaran no tener conflictos de interés.

Fuente de financiamiento: autofinanciado

Recibido: $10 / 11 / 2020$

Aceptado: 18/01/2021

Publicado: 15/02/2021

(C) Los autores. Este artículo es publicado por la revista Odontología Sanmarquina de la Facultad de Odontología, Universidad Nacional Mayor de San Marcos. Este es un artículo de acceso abierto, distribuido bajo los términos de la licencia Creative Commons Atribucion - No Comercia_Compartir Igual 4.0 Internacional. (http://creativecommons.org/licenses/by-nc-sa/4.0/) que permite el uso no comercial, distribución y reproducción en cualquier medio, siempre que la obra original sea debidamente citada. 


\section{Introducción}

El síndrome de Down (SD), fenotipo de la trisomía del par 21, es uno de los desórdenes genéticos más comunes. Afecta aproximadamente a 1 de 650 a 800 nacidos vivos en el mundo, con mayor frecuencia en niños varones (3/2), el riesgo de que la condición aparezca aumenta con la edad de la madre ${ }^{1}$. Se caracteriza por la presencia de un grado variable de discapacidad intelectual y características orofaciales específicas asociadas, como: braquicefalia, sutura metópica persistente, senos esfenoidales ausentes, senos maxilares hipoplásicos, entre otras anomalías se puede apreciar hipotelorismo ocular, nariz pequeña, aplanamiento del puente nasal, prognatismo mandibular relativo, anomalías en el odontoides, inestabilidad atlanto - axial y laxitud congénita de los ligamentos. A nivel intraoral, la lengua puede ser fisurada o geográfica, también se puede presentar macroglosia relativa o verdadera. Es común la presencia de microdoncia, hipodoncia, raíces dentales cortas y taurodontismo. Se ha reportado una relación entre el SD y la baja prevalencia de caries dental y alta prevalencia de enfermedad periodontal. Estas características junto con el desarrollo psicosomático retardado e hipotonía muscular facial, conducen a la aparición de parafunciones y disfunciones (en la succión, masticación, deglución o habla), que a su vez afectan la frecuencia de maloclusiones, observándose mordida cruzada posterior, mordida abierta anterior y respiración bucal ${ }^{2-6}$. Se ha mencionado que los niños con SD presentan retraso en la erupción dental (ED), sin embargo, no se conoce la etiología de dicho retraso ni su magnitud. Siendo que la ED es un proceso fisiológico que influencia fuertemente el desarrollo normal del complejo craneofacial ${ }^{7,8}$, el objetivo de la presente revisión fue analizar los conceptos de fisiología de la ED y acerca de la evidencia disponible respecto al retraso en la ED en niños con SD, y si ese retraso tuvo relación con problemas en la maduración dental o con otros factores sistémicos y locales.

\section{Metodología de búsqueda y selección de resultados}

Se realizó una revisión de la información sobre la fisiología de la ED, el retraso en la ED y su relación con la maduración dental en niños con SD. Para ello se incluyó información de libros de interés sobre el tema, además de una búsqueda en la base de datos bibliográficos de PubMed y ScienceDirect. Los términos de búsqueda fueron "tooth eruption", "delay tooth eruption", "dental age", "Down syndrome". No hubo restricción por antigüedad de publicación. Se obtuvo 49 artículos científicos en total que incluyeron estudios originales, artículos de revisión, reportes de casos y revisiones sistemáticas. Los resultados fueron obtenidos en idioma inglés. Finalmente se incluyeron 34 artículos científicos cuyo título y resumen respondían a los objetivos de la presente revisión. La información obtenida fue clasificada y presentada para describir los hallazgos de manera narrativa con una secuencia ordenada.

\section{Fisiología de la ED}

Se define a la ED como el proceso de desarrollo, responsable de mover un diente desde la posición en su cripta, a través del proceso alveolar, dentro de la cavidad oral hasta su posición final de oclusión con su antagonista. Es un proceso fisiológico que influencia fuertemente el desarrollo normal del complejo craneofacial. Así mismo, es un proceso dinámico que acompaña el completo desarrollo de la raíz, el establecimiento del periodonto y el mantenimiento de una oclusión funcional ${ }^{7,8}$.

Según Marks ${ }^{9}$, la etapa intraósea de la ED implica que ocurra reabsorción ósea para formar una vía de erupción y la formación de hueso interradicular, el crecimiento de la raíz y la aposición ósea que mueven el diente en erupción, incluida la reabsorción de las raíces de dientes primarios y la penetración epitelial, para que posteriormente la pieza se posicione dentro del arco dental. Estos eventos están regulados por el folículo dental que desarrolla regiones para iniciar y controlar la formación y reabsorción ósea. La formación de raíces se acomoda durante la ED y es una consecuencia del proceso, no una causa. Después de la ED, el ligamento periodontal se forma y se reorganiza siendo poco probable que contribuya sustancialmente a la ED, pero puede tener un papel al final del proceso. Ciertamente el ligamento periodontal contribuye a la estabilidad del funcionamiento del diente, y posiblemente la formación de cemento apical mantenga un movimiento eruptivo lento durante toda la vida del diente ${ }^{9}$.

En algunas de las investigaciones que se citan en esta revisión se toma el término de "emergencia dental", el cual es diferente a "ED", siendo que la "emergencia dental" sólo describe el momento en que aparece alguna parte de la cúspide o corona de la pieza dental a través de la encía, por lo tanto es frecuentemente empleado como marcador clínico de la erupción de la pieza dental ${ }^{10}$.

El tiempo de ED ha sido estudiado en humanos, tanto en la dentición primaria como en la permanente, y se ha observado que los dientes de las niñas erupcionan más pronto que en los niños ${ }^{11}$. También se ha considerado que, en circunstancias normales, la ED comienza cuando se establece 3/4 de su longitud final de raíz. Es decir, si un diente erupcionado tiene menos desarrollo radicular que los 3/4 de longitud esperados, la ED se considera prematura, mientras que, si el diente se ha desarrollado más que la longitud radicular y no se ha dado la ED, debe definirse como retraso. Sin embargo, se ha visto que, en el momento de la ED, los caninos mandibulares y los segundos molares muestran un desarrollo radicular más avanzado que los 3/4 esperados, mientras que los incisivos centrales mandibulares y los primeros molares muestran un desarrollo radicular inferior a los 3/4 esperados, cuando ya empiezan su emergencia ${ }^{8}$.

Algunos autores consideran que la pieza dental se encuentra en retraso de ED cuando su emergencia se presenta un tiempo después de dos desviaciones estándar del promedio normal establecido ${ }^{8}$. También se ha usado como criterio, la observación de la pieza homóloga 
contralateral, sin embargo, no se ha determinado cuánto tiempo debería transcurrir después de la erupción del diente correspondiente para afirmar si existe retraso de ED del homólogo en la misma arcada dental ${ }^{12}$. Un retraso en la ED representaría un impacto negativo en el tratamiento ortodóncico de una persona, afectando directamente el diagnóstico y el plan de tratamiento, ya que puede provocar variaciones en la cronología de erupción de las piezas dentales ${ }^{13}$.

\section{Etiología del retraso de la ED en SD}

Se ha tratado a las condiciones sistémicas, hormonales, raciales y nutricionales como probables influencias en la ED ${ }^{14-16}$. Entre las afecciones sistémicas reportadas de intervenir en el tiempo de ED se destacan los trastornos endocrinos de la madre, quimioterapia o medicamentos a largo plazo que pueden inhibir la vía de las prostaglandinas y disminuir la actividad osteoclástica en los tejidos periodontales, lentificando la tasa de $\mathrm{ED}^{8}$. La desnutrición crónica y prolongada, con deficiencias de vitaminas durante la infancia, también se ha correlacionado con la ED tardía ${ }^{13}$.

También se asocia el retraso de ED con factores locales como: barreras mucosas, fibromatosis gingival, tumores odontogénicos y no odontogénicos, dientes supernumerarios, anquilosis dental, quistes, erupción ectópica, pérdida prematura de un diente primario, impactación dental o daño por radiación ${ }^{8}$.

Desórdenes genéticos se han asociado con retraso de ED, como el SD, síndrome de Turner, síndrome de Gardner, disostosis cleidocraneal, displasia ectodérmica anhidrótica, síndrome de Hutchinson-Gilford, síndrome de Bloch-Sulzberger, síndrome de Apert, síndrome de Axenfeld-Rieger ${ }^{17,18}$. Se han sugerido algunos mecanismos para explicar el retraso de ED en los desórdenes genéticos como: retraso del desarrollo generalizado, anormalidades en los tejidos periodontales, deficiencias en el cemento celular, expresión inadecuada de algunas citoquinas, y densidad ósea incrementada que impide la reabsorción ${ }^{8}$. Existe evidencia sugestiva de que la tasa de ED está influenciada por la vascularización del tejido conjuntivo perirradicular. La mala circulación periférica podría ser un factor que provoque un retraso en la ED ${ }^{19}$.

La forma en que la expresión génica en los niños con SD influye en su desarrollo dental aún no se conoce completamente. Según Van der Linden, es posible que una alteración genética en el sistema intercelular proteico RANKL/RANKL/OPG (regulador de la proporción formación/resorción ósea) y/o la alteración del complejo RUNX2 (esencial en la diferenciación osteoblástica) sea una causa de la alteración de la ED ${ }^{20}$.

Existen pocos estudios en la población de niños con SD que hayan investigado acerca del retraso en la erupción de la dentición decidua y permanente. Las raíces más cortas de los individuos con SD pueden conducir a un menor desarrollo de la altura vertical de los maxilares y pueden resultar en una altura vertical relativamente corta en el perfil facial ${ }^{4}$. Algunos autores apoyan la idea de que el retraso en la ED puede ser parte del retraso en el crecimiento y desarrollo que caracteriza al SD, incluso han relacionado el bajo peso al nacer del niño con SD con el retraso de la ED ${ }^{19}$, así como la presencia de hipotiroidismo en la infancia, ya que del 14 al 50\% de ellos lo pueden presentar ${ }^{20-22}$.

Sin embargo, hay niños que no presentan alteraciones sistémicas ni locales y que también presentan retraso en la ED sin asociarse a ninguna de las causas antes mencionadas. En estos casos, se dice que la etiología podría ser alguna alteración en la regulación del proceso de ED a nivel celular ${ }^{18,23}$.

Teniendo a la "emergencia dental" como indicador clínico de la ED observamos estudios como el de Pacurar ${ }^{1}$ en niños con SD donde se observó retraso de 1,27 años en la ED, respecto a valores normales ${ }^{1}$. En Chile se realizaron dos investigaciones importantes. En la primera, se observó que la cronología del proceso eruptivo de piezas temporales en niños y niñas con SD fue diferente a la de una población sin SD. Los varones con SD registraron un retraso significativo en el momento de la erupción de las siguientes piezas dentales: incisivo central derecho superior, los incisivos laterales superiores derecho e izquierdo; el incisivo central derecho inferior y los caninos inferiores derecho e izquierdo. En las mujeres con SD se registró retraso en la ED de más piezas dentales: los incisivos laterales superiores derecho e izquierdo, los caninos superiores derecho e izquierdo, el primer molar superior izquierdo, el incisivo central inferior izquierdo, los incisivos laterales inferiores derecho e izquierdo, los caninos inferiores derecho e izquierdo y el segundo molar inferior derecho ${ }^{24}$. En el segundo estudio chileno se investigó el retraso de erupción en piezas permanentes, y observaron diferencias menores para las siguientes piezas dentales: caninos y premolares, primeras molares superiores e inferiores, así como los incisivos centrales y laterales; también observaron diferencias en cuanto a la secuencia cronológica de ED en los nińos con SD comparados con niños sin SD, notándose principalmente en caninos, y premolares. Así mismo, observaron una dependencia entre la presencia de asimetrías de ED y la edad, ya que estas asimetrías se presentaron menos entre los 7 y 9 años de edad, y más frecuentemente entre los 10 y 14 años de edad ${ }^{25}$.

Otra forma de evaluación clínica del retraso de ED en niños con SD ha sido observada en el estudio de De Moraes et al. quienes emplearon como indicador "la presencia de los otros dientes de la cavidad oral", por lo que sus comparaciones no son confiables, como es reconocido por los mismos autores de dicho estudio; mostrando un solo caso de retraso en la ED ocurrido en una mujer de 15 años con SD, en la que se formaron todos los dientes, excepto el segundo premolar superior, cuya formación de la raíz recién comenzaba ${ }^{15}$.

Se ha reportado el caso de una niña de China de 15 años con SD, quien no presentó ninguna pieza dental permanente lo que está mucho más allá del rango del tiempo de ED. El examen radiográfico reveló que la niña presentaba impactación de los dientes permanentes y agenesia 
de los segundos premolares superiores. Es importante observar que, aunque la erupción de los dientes incisivos se retrasó significativamente, el desarrollo de las raíces estaba completo. Después de la extracción de los dientes temporales correspondientes, los incisivos impactados tuvieron la probabilidad de lograr la ED espontánea ${ }^{20}$.

\section{Maduración dental en SD}

Existen escasos estudios que hayan investigado si el retraso en la ED sea resultado de una maduración dental tardía, y si esto ocurre en afecciones sindrómicas como el SD. Se entiende la maduración dental, como aquélla que comprende desde la calcificación de las coronas hasta el desarrollo radicular de las piezas dentales. Algunos autores afirman que resulta más importante saber si la maduración de la pieza dental está retrasada o no, dado que las tasas de calcificación de los dientes están más controladas por genes que por factores ambientales y que la emergencia dental representa sólo una etapa en el proceso continuo de $\mathrm{ED}^{26,27}$.

Para determinar el grado de maduración de una pieza dental se evalúan los estadios de mineralización, ya que es posible el reconocimiento de dichos estadios en las radiografías panorámicas y/o periapicales en la práctica odontológica ${ }^{26,28}$. En la actualidad se cuentan con diversos índices que permiten establecer el estadio de maduración dental en los niños, como por ejemplo los Métodos de Nolla, Sistema de Demirjian, Sistema de Atlas de London, Método de Cameriere, etc. Estos métodos se basan en la formación dentaria (desde la primera apariencia de los puntos calcificados hasta el cierre del ápice de las raíces dentales), observando la apariencia radiológica de las piezas dentales seleccionadas, otorgándoles escalas o estadíos, que varía en cada método ${ }^{29,30}$. $\mathrm{Al}$ emplear estos métodos el resultado puede arrojar la edad dental del paciente, determinándose el grado de correlación con su edad cronológica, lo cual ha sido probado en poblaciones de origen étnico diferente y con resultados no siempre coincidentes ${ }^{31,32}$.

Existen estudios de casos y controles que investigaron la maduración dental en niños con SD. Uno de ellos, empleó el método de Nolla en radiografías panorámicas de niños brasileños con SD y no encontraron diferencias entre la edades dental y cronológica de los niños sin SD. Una limitación importante de este estudio es que la evaluación estuvo restringida al segundo molar permanente y fue realizada por un solo examinador ${ }^{33}$. En otro estudio, donde también emplearon el método de Nolla en niños con SD, observaron que la edad dental fue inferior en comparación a su edad cronológica, sin embargo, la diferencia fue leve en el 33,33\% de las mujeres con SD y en $54,28 \%$ para los hombres con SD. Aunque observaron similar situación en el grupo control de niños $\sin S D^{15}$.

Van der Linden ${ }^{20}$ investigó si las anomalías en la maduración dental en niños con SD eran la causa del retraso en la ED. Usando el método de Demirjian estableció que la maduración dental en niños con SD era similar al de los niños de su grupo control.
También se ha empleado el método de Demirjian en otras poblaciones de niños con SD observando que la edad dental sobreestimó la edad cronológica en $64 \%$ de los hombres y $77 \%$ de las mujeres, es decir los resultados del método empleado calcularon una edad dental mayor a la edad cronológica, que contradijo la idea de esperar un retraso en la edad dental en la mayor parte de la muestra estudiada ${ }^{34}$.

Un estudio que empleó ambos métodos, de Nolla y de Demirjian, en individuos españoles con diversas discapacidades físicas y mentales congénitas o perinatales, 37 de ellos con SD, encontraron un retraso significativo de la edad dental en las niñas en comparación con su edad cronológica ${ }^{17}$.

La influencia de los síndromes genéticos en la maduración dental no ha podido ser determinada. En el estudio de Pinchi ${ }^{35}$ se evaluó la edad dental mediante el sistema de Atlas de Londres, en niños con síndromes genéticos variados, entre ellos el SD y encontraron que la maduración dental era similar entre niños con y sin síndromes genéticos. Empleando el mismo Atlas de Londres en radiografías panorámicas, Pereira ${ }^{36}$ estimó la edad dental de grupos de niños con necesidades especiales (incluidos niños con SD) y no observó diferencias entre sus grupos de estudio, concluyendo que este atlas puede ser potencialmente utilizado como una herramienta para la estimación de la edad en niños con diversos síndromes genéticos .

En niños brasileños con SD se empleó un programa informático para evaluar estadios de mineralización dental, el cual definió las diferencias entre la edad dental y edad cronológica así: diferencias de 3 meses no son significativas; diferencias de hasta 12 meses son consideradas levemente adelantadas o retrasadas, aunque son consideradas dentro de los estándares normales; diferencias por arriba de 12 meses se consideran fuera de los estándares normales y diferencias de edad de más de 24 meses se consideran muy fuera de los estándares normales. En dicho estudio, la mayoría de los pacientes con $\mathrm{SD}$ se consideraron dentro de los estándares normales en la cronología de mineralización dental, dos tercios de los niños y niñas presentaron edad dental con diferencias de hasta 12 meses, y sólo el $18,87 \%$ de los hombres y el $10,21 \%$ de las mujeres presentaron edad dental fuera de los estándares normales, con diferencias de más de 24 meses ${ }^{37}$.

\section{Discusión}

Los síndromes cromosómicos, como el SD, que surgen de defectos numéricos y estructurales de los cromosomas a menudo incluyen manifestaciones que afectan la región craneofacial e incluyen manifestaciones orales características, como agenesias dentales múltiples u otras condiciones que pueden modificar el desarrollo dental esperado ${ }^{36}$ y que pueden estar asociados a retardo en el desarrollo psicosomático ${ }^{15}$. Una manifestación a nivel oral es la presencia de raíces dentales cortas, aunque no está claro si ello influye en la ED ${ }^{4}$. Otra condición a considerar es que del 14 al $50 \%$ de los pacientes con 
SD pueden presentar hipotiroidismo, del cual se conoce su relación con el retraso en la ED ${ }^{22,23}$. Así mismo, la hipotonicidad de la musculatura facial presente en el $\mathrm{SD}$, conduce a complicaciones en el desarrollo de la succión, la masticación, la deglución y el habla; el grado de dificultad experimentado por cada paciente con SD es variable 4,38 .

Existe una escasa evidencia científica acerca de las causas del retraso en la ED de los niños con SD. Esto puede deberse a que, esencialmente, tampoco se tiene suficiente información sobre los factores que controlan el proceso eruptivo normal. En la presente revisión se han incluido las investigaciones encontradas respecto a la ED en los niños con SD, que han buscado determinar el tiempo exacto de la "emergencia dental", mediante la evaluación clínica de las piezas dentales, y aquellas investigaciones que estudiaron si la maduración dental puede influir en el retraso de la ED en niños con SD ${ }^{11}$.

De los estudios que detectan clínicamente la "emergencia dental", los que destacan son los de Ondarza ${ }^{24}$ y Jara ${ }^{25}$, quienes sostienen que los niños con SD necesitan más tiempo para completar su dentición decidua y permanente en comparación a los niños sin $\mathrm{SD}$, sin embargo, el retraso en la ED no se dio en todas las piezas dentales en los niños de su muestra. Ya hemos definido que el acto de observar la "emergencia dental" puede estar sometida a factores locales, además de las anomalías y agenesias dentales que pueden presentar los niños con $\mathrm{SD}$, es por eso que resulta importante que estos estudios clínicos definan adecuadamente su metodología y describan los hallazgos individuales relevantes.

Las investigaciones que plantearon la hipótesis de que la maduración dental tardía podría ser la causa del retraso en la ED, prefirieron evaluar la pieza dental radiográficamente y guiarse del grado de mineralización en las radiografías panorámicas y/o periapicales. $\mathrm{Al}$ darle más importancia al proceso de maduración dental, emplearon diversos índices para identificar el grado de maduración de las piezas dentales y así determinar la edad dental, relacionarla con la edad cronológica, y observar el grado de concordancia entre ambas 15,17,21,33-37. Se han empleado métodos muy distintos para determinar la maduración dental, cada uno de ellos con sus propios criterios de evaluación, por tal motivo, resulta difícil comparar algunos resultados, ya que en ciertos casos el investigador restringe su análisis a algunas piezas dentales, como lo hizo De Moraes ${ }^{33}$, lo que podría explicar la subestimación o sobreestimación de la edad dental, incluso hay que considerar el sesgo que podría surgir cuando las radiografías son analizadas por un solo examinador. Así también, se debe considerar que los métodos de maduración dental como el de Nolla y Demirjian fueron realizados en poblaciones muy distintas en las que se aplican. También, mencionar que en las investigaciones que forman parte de esta revisión, se han mostrado leves retrasos de la edad dental en niños con SD como son los de Hala ${ }^{14}$, Diz ${ }^{16}$ y De Moraes ${ }^{37}$ y otros no encontraron diferencias importantes entre la edad dental y la edad cronológica, allí tenemos a Van der
Linden ${ }^{20}$, Pinchi ${ }^{35}$, y Pereira ${ }^{36}$, hasta incluso se halló en el estudio de Bagattoni ${ }^{34}$ que la edad dental estuvo sobreestimada en relación a la edad cronológica, es decir la madurez dental estuvo adelantada a la edad cronológica que le correspondía. Es por eso que investigadores como Hala ${ }^{14}$ afirman que "se requiere más precaución para la estimación de la edad de las personas con SD, ya que presentan mucha más variación que las personas $\sin \mathrm{SD}^{\prime \prime}$.

A pesar de dichas variaciones, la mayoría de los estudios revisados concluyen que la maduración dental en sujetos con y sin SD no es diferente y que el retraso en la ED en la cavidad bucal es probablemente debido a un desarrollo dental tardío o a otros procesos que tienen lugar durante la $\mathrm{ED}^{21}$. De este modo, se apoyan los hallazgos que indican que el sistema dental es muy confiable en los procedimientos de estimación de la edad cronológica, ya que la maduración dental se considera muy estable, apenas afectada por alteraciones como los síndromes genéticos, y por factores ambientales y nutricionales en comparación con el análisis de la maduración esquelética, que al parecer puede ser más afectada ${ }^{35}$.

Por lo tanto, descartando la hipótesis que la maduración dental tardía pueda afectar la ED en niños con $\mathrm{SD}$, se enuncia otras probables causas como lo mencionado por Van der Linden ${ }^{20}$ quien indica que el retraso en la "emergencia clínica" probablemente tiene su origen en el tejido gingival u otro factor como los procesos celulares en el lado apical y oclusal del diente en erupción. Además, también indica que este retraso en la ED se le ha atribuido al retraso en el crecimiento y desarrollo del maxilar y la mandíbula, elementos característicos del SD.

Debido a que la ED es un proceso fisiológico que tiene una influencia significativa en el desarrollo normal del complejo orofacial, un retraso en la ED puede influir en la precisión del diagnóstico y en el plan de tratamiento, por tanto, puede tener un impacto significativo en el estado de salud del paciente, es por eso que los niños con SD requieren un seguimiento prolongado para interceptar cualquier anomalía relacionada con el período de erupción de sus dientes con el fin de aumentar su calidad de vida y mejorar su estado de salud bucal.

\section{Conclusiones}

Es difícil establecer los mecanismos responsables de la $\mathrm{ED}$, debido a que la naturaleza del proceso eruptivo es compleja y existen pocas descripciones de casos, lo cual genera poca evidencia para entender mejor este tema. Sin embargo, con los resultados observados en los estudios revisados, concluimos que no existe retraso en la maduración dental en niños con SD, y que al parecer no sería una causa del retraso en la ED y que algunas variaciones observadas en la determinación de la maduración dental en los diversos estudios presentados, pueden ser debido a que las poblaciones donde se aplicaron fueron distintas o deberse a errores en la metodología empleada. De presentarse retraso en la ED de los dientes deciduos o permanentes podría ser parte del retraso general 
del crecimiento y del desarrollo que caracteriza al SD. Se sugiere realizar estudios clínicos longitudinales con índices específicos que permitan su reproducibilidad para el reconocimiento del patrón de ED en niños con SD.

\section{Referencias bibliográficas}

1. Pacurar M, Dragomir B, Szalontay AS, Romanec C. Orthodontic Aspects on the Chronological and Dental Age in Children with Down Syndrome. Rev Chim. 2018,69(1):208-13.

2. Gorlin RJ, Cohen MM, Hennekam RC. Syndromes of the Head and Neck. En: Chapter 3 Chromosomal Syndromes Common and/or Well-Known Syndromes. 4ta edición. Edit: Oxford University Press. 2010:35-39.

3. Siqueira, WL, Nicolau J. Stimulated whole saliva components in children with Down syndrome. Spec Care Dentist. 2002;22(6):226-30. DOI: 10.1111/j.17544505.2002.tb00276.x

4. Hennequin M, Faulks D, Veyrune JL, et al. Significance of oral health in persons with Down syndrome: a literature review. Dev Med Child Neurol. 1999;41:275-83. DOI: $10.1017 / \mathrm{s} 0012162299000596$.

5. Culebras E, Silvestre J, Silvestre FJ. Alteraciones odonto-estomatológicas en el niño con síndrome de Down E. Rev esp pediatr. 2012;68(6):434-9.

6. Pérez DA. Síndrome de Down. Rev Act Clin. 2014;45:2357-61.

7. Schour I, Massler M. Studies in tooth development: growth pattern of the human teeth. Part I. J Am Dent Res. 1940;27:1778-93.

8. Suri L, Gagari E, Vastardis H. Delayed tooth eruption: pathogenesis diagnosis, and treatment. A literature review. Am J Orthod Dentofacial Orthop. 2004;126(4):432445. DOI: 10.1016/j.ajodo.2003.10.031

9. Marks SC, Schroeder HE. Tooth eruption: Theories and facts. Anat Rec.1996;245(2):374-393. DOI: 10.1002/ (SICI) 1097-0185(199606)245:2<374::AID-AR18>3.0. $\mathrm{CO} ; 2-\mathrm{M}$

10. Gorski J, Marks, SC. Current Concepts of the Biology of Tooth Eruption. Crit Rev Oral Biol Med. 1992;3(3):185206. DOI:10.1177/10454411920030030201

11. Kjær I. Mechanism of Human Tooth Eruption: Review Article Including a New Theory for Future Studies on the Eruption Process. Scientifica (Cairo). 2014;2014:341905. DOI: 10.1155/2014/341905.

12. Makino E, Tsujino K, Ishii T, Shintani S, Sueishi K. Difference in Bilateral Timing of Eruption of Permanent Teeth. Bull Tokyo Dent Coll. 2018;59(4):277-84. DOI:10.2209/tdcpublication.2018-0009

13. Vaillard E, Huitzil E, Moyaho MA, Ortega A, Castillo L. Efectos de la desnutrición infantil en la erupción dental. Rev Tamé. 2015;3(9):289-96.

14. Hala LA, Moraes ME, Carvalho MF, Lopes SL, Gamba TO. Comparison of accuracy between dental and skeletal age in the estimation of chronological age of Down syndrome individuals. Forensic Sci Int. 2016;266:578. e1-578.e10. DOI:10.1016/j.forsciint.2016.06.019
15. De Moraes ME, De Moraes LC, Dotto GN, Dotto PP, Dos Santos LR. Dental Anomalies in Patients with Down Syndrome. Braz Dent J. 2007;18(4):346-50. DOI: $10.1590 / s 0103-64402007000400014$

16. Diz P, Limeres J, Salgado AFP, Tomás I, Delgado LF, Vásquez E, et al. Correlation between dental maturation and chronological age in patients with cerebral palsy, mental retardation, and Down syndrome. Res Dev Disabil. 2011;32(2):808-17. DOI: 10.1016/j.ridd.2010.10.019

17. Jain P, Rathee M. Anatomy, Head and Neck, Tooth Eruption. [Updated 2019 Nov 6]. In: StatPearls [Internet]. Treasure Island (FL): StatPearls Publishing; 2020.

18. Javadinejad S, Sekhavati H, Ghafari R. A Comparison of the Accuracy of Four Age Estimation Methods Based on Panoramic Radiography of Developing Teeth. J Dent Res Dent Clin Dent Prospect. 2015;9(2):72-8. DOI: 10.15171/joddd.2015.015

19. Wang Y, Zhao Y, Ge L. Delayed Eruption of Permanent Teeth in an Adolescent With Down's Syndrome: A Case Report. J Med Cases. 2015;6(6):277-8. DOI: http://dx. doi.org/10.14740/jmc2166w

20. Van der Linden MS, Vucic S, Van Marrewijk DJ, Ongkosuwito EM. Dental development in Down syndrome and healthy children: a comparative study using the Demirjian method. Orthod Craniofac Res. 2017;1-6. DOI: $10.1111 /$ ocr.12139

21. Reynoso ME, Monter MA, Sánchez I. Hipotiroidismo congénito y sus manifestaciones bucales. Rev Odontol Mex. 2014;18(2):132-7.

22. AlAaraj N, Soliman AT, Itani M, Khalil A, De Sanctis V. Prevalence of thyroid dysfunctions in infants and children with Down Syndrome (DS) and the effect of thyroxine treatment on linear growth and weight gain in treated subjects versus DS subjects with normal thyroid function: a controlled study. Acta Biomed. 2019;90, Suppl 8:36-42. DOI: 10.23750/abm.v90i8-S.8503

23. Cuadros C, Rubert A, Guinot F, Bellet J. Etiología del retraso de la erupción dental: revisión de literatura. Dentum. 2008;8(4):155-66.

24. Ondarza A, Jara L, Muñoz P, Blanco R. Sequence of eruption of deciduous dentition in a Chilean sample with Down's syndrome. Arch Oral Biol. 1997;42(5):4016. DOI: 10.1016/s0003-9969(97)00012-5

25. Jara L, Ondarza A, Blanco R, Valenzuela C. The sequence of eruption of the permanent dentition in a Chilean sample with Down's syndrome. Arch Oral Biol. 1993;38(1):85-9. DOI: 10.1016/0003-9969(93)90160-n

26. Uysal T, Sari Z, Ramoglu S, Basciftci F. Relationships Between Dental and Skeletal Maturity in Turkish Subjects. Angle Orthod. 2004;74(5):657-64. DOI: 10.1043/0003-3219(2004)074<0657:RBDASM>2.0. $\mathrm{CO} ; 2$

27. Manjunatha, B., \& Nishit, K. Estimation of age from development and eruption of teeth. J Forensic Dent Sci. 2014;6(2):73-6. DOI: 10.4103/0975-1475.132526

28. Cameron A, Widmer R. Manual de odontología pediátrica. Cap 9. Anomalías dentales. 3ra ed. Harcourt Brace. España: 2010 
29. Nolla C. The development of the permanent teeth. J. Dent Child. 1960;27(4):254-66.

30. Demirjian A, Goldstein H, Tanner J. A new system of dental age assessment. Hum Biol. 1973;45(2)211-227.

31. Nur B, Kuzgoz A, Bayram M, Celikoglu M, Nur M, Kayipmaz S, Yildirim S. Validity of Demirjian and Nolla methods for dental age estimation for Northeastern Turkish children aged 5-16 years old. Med Oral Patol Oral Cir Bucal. 2012;17(5): e871-e877. DOI: 10.4317/ medoral. 18034

32. Cruz A, Linares J, Martínez M, Rodríguez M, Otero X, Concheiro L. Dental age estimation in Spanish and Venezuelan children. Comparison of Demirjian and Chaillet's scores. Int J Legal Med. 2010;124(2):105-12. DOI: $10.1007 / \mathrm{s} 00414-009-0380-5$

33. De Moraes ME, De Moraes LC, Cardoso M, Ursi W, Lopes SL. Age assessment based on dental calcification in individuals with Down syndrome. Res Dev Disabil. 2013;34(11):4274-9. DOI: 10.1016/j.ridd.2013.09.005

34. Bagattoni S, D'Alessandro G, Gatto MR, Piana G. Applicability of Demirjian's method for age estimation in a sample of Italian children with Down syndrome: A case-control retrospective study. Forensic Sci Int. 2019;298:336-40. DOI: 10.1016/j.forsciint.2019.03.015
35. Pinchi V, Bugelli V, Vitale G, Pradella F, Farese L, Focardi M. Dental age estimation in children with chromosomal syndromes. J Forensic Odontostomatol. 2018;36(1): 44-52

36. Pereira CP, Russell LM, Fernandes MP, Da Silva RH, Filipe R, Santos VS. Dental Age Estimation based on Development Dental Atlas Assessment in a Child/Adolescent Population with Systemic Diseases. Acta stomatol Croat. 2019;53(4):307-17. DOI: 10.15644/asc53/4/1

37. De Moraes ME, Bastos M, Santos LR, Castilho JC, De Moraes LC, Filho EM. Dental age in patients with Down syndrome. Braz Oral Res. 2007;21(3):259-64. DOI: $10.1590 / \mathrm{s} 1806-83242007000300012$.

38. Glass TJ, Twadell SL, Valmadrid LC, Connor NP. Early impacts of modified food consistency on oromotor outcomes in mouse models of Down syndrome. Physiol Behav. 2019;199:273-81. DOI: 10.1016/j.physbeh.2018.11.031 
